\title{
Antitumor Immunity in Agaricus sp., Paffia paniculata, and Propolis
}

\author{
Yeunhwa Gu, ${ }^{1}$ Takenori Yamashita, ${ }^{1}$ Masami Oshima, ${ }^{1}$ Ikukatsu Suzuki, ${ }^{1}$ \\ Toshibiro Maenaka, ${ }^{1}{ }^{\mathcal{\sigma}}$ Noribide Mitsumoto ${ }^{2}$ \\ ${ }^{1}$ Faculty of Health Science, Suzuka University of Medical Science, 1001 Kishioka-cho Suzuka \\ Mie 510-0293, Japan; ${ }^{2}$ Shizen Kyusei Co., Ltd, Okayama, Japan
}

We investigated the antioxidant activity, lymphocyte versus polymorphonuclear leukocytes enhancement activity (L/P activity), and antitumor activity of three simple substances (Agaricus sp., Paffiapaniculata, and Propolis) as well as a mixture of these three kinds of substances (ABP).

A powdered mixture $(\mathrm{ABP})$ of these three substances were prepared using an agate mortar. For the extraction, $2000 \mathrm{~mL}$ of water was added to $300 \mathrm{~g}$ of finely powdered Agaricus sp. and then stirred for 2 hours in a water bath of $40^{\circ} \mathrm{C}$. After centrifugation for 10 minutes at $5000 \mathrm{rpm}$, the supernatant was filtered using folded filter paper. Distilled water $(2000 \mathrm{~mL})$ was added to the precipitates, and extraction was repeated in the same way. Dried Agaricus sp. was obtained by combining the supernatant from the first extraction with the supernatant from the second extraction (yield: 48.0 g, recovery: 16\%). Propolis (100 g) was powdered, and $300 \mathrm{~mL}$ of $70 \%$ ethanol was added. After drying at room temperature, filtration was carried out using folded filter paper. The filtrates were dried using an evaporator. Dried Propolis was obtained by freezing and thawing (yield: $53.5 \mathrm{~g}$, recovery: $53.5 \%$ ). Distilled water $(2000 \mathrm{~mL})$ was added to $200 \mathrm{~g}$ of finely powdered Paffia paniculata. Dried P.paniculata was obtained by the same technique (yield: $83.6 \mathrm{~g}$, recovery: $41 \%)$.

With respect to radical scavenging activity, water and P.paniculata had no radical scavenging activity and showed no marked difference, whereas Agaricus sp. showed slight radical scavenging activity. Both $\mathrm{ABP}$ and Propolis showed greater radical scavenging activity than $0.2 \mathrm{mM}$ Trolox, which was used as a positive standard.

Ten neonatal Swiss-Webster mice were divided into two groups. Saline was injected intraperitoneally into one group, and Agaricus sp. was injected intraperitoneally into the other group at a dose of $200 \mu \mathrm{g} /$ mouse. $\mathrm{L} / \mathrm{P}$ ratios after the administration of ABP $(200 \mu \mathrm{g} /$ mouse) were $0.91 \pm 0.07$ (day 6), $3.23 \pm 0.39$ (day 10), and $4.82 \pm 0.46$ (day 14), whereas $\mathrm{L} / \mathrm{P}$ ratios in the control group were $0.43 \pm 0.04$ (day 6), $0.96 \pm 0.08$ (day 10 ), and $1.43 \pm 0.39$ (day 14). The $\mathrm{L} / \mathrm{P}$ ratio after the administration of Propolis was significantly elevated $(p<0.01)$, as compared to the control group.

When the ABP mixture was administered at a dose of $400 \mu \mathrm{g} / \mathrm{kg}$ for 34 consecutive days, remarkably high antitumor activity (suppressive ratio: $85.1 \%, p<0.01$ ) was shown.

In Sarcoma 100 solid carcinoma, when Agaricus sp. (200 mg/kg B.W./day), Paffia paniculata $(60 \mathrm{mg} /$ $\mathrm{kg}$ B.W./day), and Propolis (80 mg/kg B.W./day) were orally administered for 34 consecutive days, suppressive ratios were $60.3 \%(p<0.05), 54.8 \%$ $(p<0.05)$, and 62.6\% $(p<0.05)$, respectively. When the ABP mixture was administered at a dose of 400 $\mathrm{mg} / \mathrm{kg}$ B.W./day for 34 consecutive days, remarkably high antitumor activity (suppressive ratio: $83.5 \%$, $p<0.01)$ was shown. 